Wien klin Mag 2020 · 23:1

https://doi.org/10.1007/s00740-020-00331-7

(c) Springer-Verlag GmbH Austria, ein Teil von Springer Nature 2020

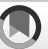

Verena Kienast

SpringerMedizin, Wien, Österreich

\title{
Grenzenlos, rücksichtslos und sinnlos
}

\section{Wenn Streben zur Gier wird}

Immer noch mehr zu wollen, ist in unseren westlichen Gesellschaften zu einem menschlichen Charakteristikum geworden. Das ist an sich nichts Schlechtes. Wenn dieses Streben allerdings ohne Rücksicht auf Verluste und zum anhaltenden Nachteil anderer verfolgt wird, wenn keine Grenzen oder eine gewisse Sättigung mehr wahrgenommen wird, wird die Sache bedenklich bis gefährlich. Nicht umsonst zählt die Gier zu einer der sieben Todsünden, wie sie die Bibel als Gegensätze der sieben Tugenden nennt, wobei hier die Gier als Habsucht spezifiziert wird. Mit diesem Begriff der "Sucht" wird sehr anschaulich das übermäßige, grenzenlose und letztlich auch sinnlose Verlangen verdeutlicht, von dem sich der Betroffene selbst nur schlecht befreien kann. Und er richtet damit Schaden an, materiell ebenso wie sozial und emotional.

\section{Zynische Alternativen}

Was die eigene Gesundheit betrifft, zeigen sich zweifellos in den sogenannten Wohlstandserkrankungen die Auswirkungen der Gier ganz unmittelbar: Zuviel Nahrung, zuviel Alkohol, Nikotin. Der absehbare Schaden ist bekannt, das Verhalten ändert sich nicht. Im größeren Zusammenhang hat die Gier im Sinne der Habsucht in unserer Gesellschaft die Unterschiede im wirtschaftlichen Wohlstand in den vergangenen Jahrzehnten vergrößert. Während manches Vermögen satt ansteigt und manisch um Million und Million vergrößert wird, übersteigen bei vielen Menschen die Lebenshal- tungskosten zunehmend das Erwerbseinkommen. Wenn Wohnen zum Luxus wird und als Alternative Kleinstwohnungen angeboten werden, die freilich den Investoren satte Renditen bringen, dann ist das nichts anderes als zynisch. Die Gier ist grenzenlos und sie lässt sich offenbar nicht durch politische Vorgaben eindämmen. Wer hat, möchte gerne mehr - auch wenn er gar nicht mehr braucht und nicht wirklich weiß, was er damit tun soll. Außer es zu haben. Aber so wie der Körper die Folgen der Gier bei der Nahrungsaufnahme und beim Alkoholkonsum irgendwann in Form von Krankheit zu spüren bekommt, kann auch die Gesellschaft deutliche Reaktionen zeigen. Es ist eine Frage der Zeit. Das neue Jahrzehnt der 20er Jahre könnte Anregung für eine spannende Rückschau auf das vergangene Jahrhundert geben ... Ob es einen Lerneffekt gegeben hat, wird sich zeigen.

\section{Auch Neugier kann überschießen}

Von einer anderen „Gier“ profitiert freilich der Fortschritt in der Welt: Der Neugier. Sie ist ein wesentlicher Bestandteil des Lebens und ermöglicht erst die geistige Entwicklung. Kinder, denen es nicht möglich ist, ihre Umwelt zu erkunden und also ihre Neugierde zu befriedigen, zeigen deutliche Defizite, sowohl kognitiv als auch sozial. Neugierde ist eine der treibenden Kräfte jeder Forschung. Aber auch hier gilt: Grenzenlose, rücksichtslose und bedenkenlose Forschung richtet Schaden an.
Ein Zuviel der Neugierde kann ebenso zur Sucht werden, wie die materielle Gier. Entsprechende Selbstreflexion wäre hier wie dort hilfreich

meint Ihre

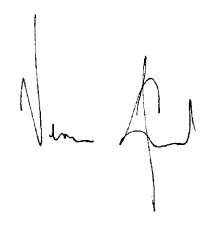

\section{Korrespondenzadresse}

Verena Kienast

SpringerMedizin

Wien, Österreich

Verena.kienast@springer.at

Interessenkonflikt. V. Kienast gibt an, dass kein Interessenkonflikt besteht.

Hinweis des Verlags. Der Verlag bleibt in Hinblick auf geografische Zuordnungen und Gebietsbezeichnungen in veröffentlichten Karten und Institutsadressen neutral. 